\title{
Striatal dopamine synthesis capacity in autism spectrum disorder and its relation with social defeat: an $\left[{ }^{18} \mathrm{~F}\right]$-FDOPA PET/CT study
}

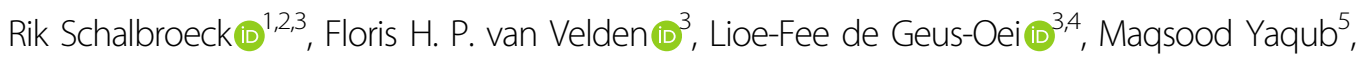
Therese van Amelsvoort ${ }^{2}$, Jan Booij ${ }^{6}$ and Jean-Paul Selten ${ }^{1,2}$

\begin{abstract}
Alterations in dopamine signalling have been implied in autism spectrum disorder (ASD), and these could be associated with the risk of developing a psychotic disorder in ASD adults. Negative social experiences and feelings of social defeat might result in an increase in dopamine functioning. However, few studies examined dopamine functioning in vivo in ASD. Here we examine whether striatal dopamine synthesis capacity is increased in ASD and associated with social defeat. Forty-four unmedicated, non-psychotic adults diagnosed with ASD and 22 matched controls, aged 18-30 years, completed a dynamic 3,4-dihydroxy-6-[ $\left.{ }^{18} \mathrm{~F}\right]-$ fluoro-L-phenylalanine positron emission tomography/computed tomography $\left(\left[^{18} \mathrm{~F}\right]-\mathrm{FDOPA}\right.$ PET/CT) scan to measure presynaptic dopamine synthesis capacity in the striatum. We considered unwanted loneliness, ascertained using the UCLA Loneliness Scale, as primary measure of social defeat. We found no statistically significant difference in striatal dopamine synthesis capacity between ASD and controls $\left(F_{1,60}=0.026, p=0.87\right)$. In ASD, striatal dopamine synthesis capacity was not significantly associated with loneliness ( $\beta=0.01, p=0.96)$. Secondary analyses showed comparable results when examining the associative, limbic, and sensorimotor sub-regions of the striatum (all $p$-values $>0.05$ ). Results were similar before and after adjusting for age, sex, smoking-status, and PET/CT-scanner-type. In conclusion, in unmedicated, non-psychotic adults with ASD, striatal dopamine synthesis capacity is not increased and not associated with social defeat.
\end{abstract}

\section{Introduction}

Adults with autism spectrum disorder (ASD) are at an increased risk of developing a psychotic disorder ${ }^{1}$. However, risk factors and neurobiological alterations associated with this risk remain poorly understood ${ }^{2}$.

A well-replicated finding of neurotransmitter functioning in psychosis has been that of increased presynaptic dopamine functioning. In vivo molecular imaging studies using positron emission tomography (PET) or singlephoton emission computed tomography (SPECT) have consistently shown that psychosis is strongly related to

\footnotetext{
Correspondence: Rik Schalbroeck (rschalbroeck@gmail.com)

${ }^{1}$ Rivierduinen Institute for Mental Healthcare, Leiden, The Netherlands

${ }^{2}$ School for Mental Health and Neuroscience, Maastricht University, Maastricht, The Netherlands

Full list of author information is available at the end of the article
}

increased presynaptic dopamine synthesis capacity and endogenous dopamine release in the striatum ${ }^{3}$. This increase in presynaptic dopamine functioning has also been reported in individuals at-risk of psychotic disorder $^{4,5}$ and in individuals with a non-psychotic disorder when psychotic symptoms are present ${ }^{6}$.

Several researchers have suggested that alterations in dopamine functioning exist in $\mathrm{ASD}^{7}$. For instance, this has been implied in theoretical work ${ }^{8}$, gene studies ${ }^{9}$, and studies examining dopamine-modulating medication ${ }^{10,11}$. However, molecular imaging studies, in particular of presynaptic dopamine, are lacking ${ }^{12}$. No studies examined striatal dopamine release, and only two studies used 3,4dihydroxy-6- $\left[{ }^{18} \mathrm{~F}\right]$-fluoro-L-phenylalanine $\left(\left[{ }^{18} \mathrm{~F}\right]\right.$-FDOPA $)$ PET to measure dopamine synthesis capacity. The first ${ }^{13}$ found no significant increase in striatal dopamine

\section{(c) The Author(s) 2021}

(c) (i) Open Access This article is licensed under a Creative Commons Attribution 4.0 International License, which permits use, sharing, adaptation, distribution and reproduction c. in any medium or format, as long as you give appropriate credit to the original author(s) and the source, provide a link to the Creative Commons license, and indicate if changes were made. The images or other third party material in this article are included in the article's Creative Commons license, unless indicated otherwise in a credit line to the material. If material is not included in the article's Creative Commons license and your intended use is not permitted by statutory regulation or exceeds the permitted use, you will need to obtain permission directly from the copyright holder. To view a copy of this license, visit http://creativecommons.org/licenses/by/4.0/. 
synthesis capacity in autistic children $(n=14)$ relative to non-autistic peers $(n=10)$, but most $(n=11)$ autistic children were sedated using propofol which can influence dopamine concentrations ${ }^{14}$. The second ${ }^{15}$ obtained evidence of increased striatal dopamine synthesis capacity in adults with Asperger syndrome $(n=8)$ relative to controls $(n=5)$, but the number of participants was small and the influence of possible risk factors was not examined.

Evidence indicates that psychosocial stress can influence dopamine functioning ${ }^{16}$. According to the social defeat hypothesis of schizophrenia, the long-term subjective experience of outsider status or subordinate position leads to increased baseline activity and/or sensitization of the brain dopamine system ${ }^{17-19}$. In line with this hypothesis, increased presynaptic dopamine functioning has been reported in non-psychotic groups presumed to be more socially defeated, such as immigrants, individuals with severe hearing impairment, and individuals with a history of childhood trauma ${ }^{20-23}$. Whether social defeat also increases presynaptic dopamine functioning in individuals with ASD, who are widely exposed to defeating experiences such as loneliness ${ }^{24}$, bullying victimization ${ }^{25,26}$, and discrimination ${ }^{27}$, remains unknown.

Here we examine striatal presynaptic dopamine synthesis capacity, measured with $\left[{ }^{18} \mathrm{~F}\right]$-FDOPA PET/computed tomography (PET/CT), in non-psychotic adults with ASD and controls aged 18-30 years. We expect (hypothesis 1a) that striatal dopamine synthesis capacity is increased in ASD relative to controls. Furthermore, we expect (hypothesis 1b) that in ASD, striatal dopamine synthesis capacity is positively associated with loneliness, as an approximate measure of social defeat. As secondary outcomes, we expect (hypothesis 2a) that adults with ASD report more social defeat than controls, and (hypothesis $2 \mathrm{~b})$ that striatal dopamine synthesis capacity is positively related to other measures of social defeat (besides loneliness) and to subclinical psychotic symptoms. Finally, we expect (hypothesis 3) the strongest associations with dopamine synthesis capacity in the associative striatum, since the largest striatal dopaminergic disruptions in psychosis have been reported in this sub-region ${ }^{3}$ (with reference to hypotheses $1 \mathrm{a}-\mathrm{b}$ and $2 \mathrm{~b}$ ).

\section{Materials and methods Participants}

ASD and control participants were Dutch adults aged 18-30 years, recruited via online social media and poster advertisements. Adults with ASD were also recruited at mental healthcare institutes, ASD housing services, university counselling services, and ASD-related websites. All of these adults had previously been diagnosed with an ASD by a registered mental health clinician, and the first author confirmed this diagnosis using the Autism Diagnostic Observation Schedule-2 (ADOS-2) module $4^{28,29}$.
The ASD and control samples consisted of 45 and 24 participants, respectively, which were frequency-matched on age, sex, and smoking-status (yes/no).

Exclusion criteria (explained in detail in Supplement 1) included a lifetime diagnosis of psychotic or bipolar disorder assessed by self-report, psychotic symptoms indicative of psychotic disorder assessed with the Comprehensive Assessment of At-Risk Mental States

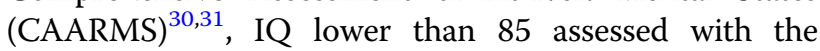
Dutch Adult Reading Test ${ }^{32}$, or a history of alcohol- or drug abuse or dependence assessed with the Composite International Diagnostic Interview (CIDI) v2.1 ${ }^{33}$. Additional exclusion criteria included self-reported or suspected neurological disorder, brain damage, history of meningitis, fragile X syndrome, Rett syndrome, 22q11 deletion syndrome, metal objects in or around the body, participation in a scientific examination where radiation was used in the past year, and (in females) lactation or pregnancy (confirmed with a urinary pregnancy test). Current and/or recent medication or illicit drug use, assessed through self-report, was also prohibited. A urinary test on the day of the PET/CT-scan had to be negative for opiates, cocaine, cannabis, and amphetamines.

\section{Sample size calculations}

Sample size calculations were conducted using G*Power $\mathrm{v} 3.1^{34,35}$, on the basis of a two-tailed alpha of 0.05 and analysis within the general linear model. The necessary sample size to examine group differences in striatal dopamine synthesis capacity was calculated using a study that compared dopamine synthesis capacity in striatal regions of adults with Asperger syndrome and controls ${ }^{15}$. If results from our study would have been similar to the smallest effect and the largest standard deviation (SD) of dopamine synthesis capacity values of that study (difference in means $=0.34, S D=0.35$ ), we would need between 19 and 24 participants assuming a power of $0.80-0.90$. Therefore, we included 24 controls.

The necessary sample size to examine the relationship between striatal dopamine synthesis capacity and loneliness in ASD was based on a study that examined the relationship between dopamine functioning and reports of childhood trauma in healthy volunteers ${ }^{21}$. Assuming a similar effect size of approximately $r=0.4$ and a power of 0.80 , we would need 45 participants. Therefore, the ASD sample included 45 participants.

\section{Design and procedures}

Participants were assessed on three separate testing days, during which they were screened for in- and exclusion criteria, underwent a magnetic resonance imaging (MRI) scan (see below), and completed measures of social defeat and an $\left[{ }^{18} \mathrm{~F}\right]$-FDOPA PET/CT-scan. All 
participants signed informed consent prior to the start of the study. The study was approved by the medical ethics committee of Leiden University Medical Center (reference NL54244.058.15), and pre-registered within the Netherlands Trial Register (registration number NL6207, https://www.trialregister.nl/trial/6207).

\section{Measures of social defeat and psychotic symptoms}

Measures of social defeat and psychosis are described in Supplement 2. In brief, we assessed loneliness using the UCLA Loneliness Scale $^{36}$ and used this as the primary measure of social defeat since it reflects a lack of social participation as well as the negative experience of this, in line with the definition of social defeat ${ }^{19}$.

As secondary measures of social defeat we assessed experiences of being ostracized using the Ostracism Experience Scale $(\mathrm{OES})^{37}$, bullying victimisation (yes/no) and its total duration before age 17 using a modified version of the Olweus Bullying Interview ${ }^{38}$, social network size using the Lubben Social Network Scale (LSNS) ${ }^{39}$, and childhood trauma before age 17 using the Childhood Trauma Questionnaire (CTQ) $)^{40,41}$. Moreover, we assessed the desire for social acceptance and belonging using the Need to Belong Scale (NBS) ${ }^{42}$, and the perceived availability of social support using the Interpersonal Support Evaluation List $(\mathrm{ISEL})^{43}$. We calculated the interaction between the NBS and ISEL and used this as a predictor variable in the analyses to examine whether a discrepancy between them was related to the outcomes.

We assessed self-reported psychotic symptoms using the Prodromal Questionnaire-16 (PQ-16) ${ }^{31,44}$. The PQ-16 was added to the project when data collection had already been started and was therefore completed by a subset of 48 participants ( $n=31$ ASD, $n=17$ controls). Furthermore, we assessed depressed mood and anxiety using the Beck's Depression Inventory-II (BDI-II) ${ }^{45}$ and State-Trait Anxiety Inventory-Trait subscale $(\mathrm{STAI}-\mathrm{T})^{46}$, which we used in exploratory analyses.

\section{MRI and PET/CT acquisition}

A structural T1-weighted MRI (3D fast field echo sequence) was obtained for each participant on a $3 \mathrm{~T}$ Ingenia MR scanner (Philips Healthcare, Best, The Netherlands). Due to a necessary replacement of the PET/ CT-scanner at the hospital in which this study was conducted, PET/CT data were collected on two different PET/CT systems, i.e. a Biograph Horizon with TrueV option (Siemens Healthineers, Erlangen, Germany) or Vereos (Philips Healthcare, Best, The Netherlands). Both systems received EARL PET/CT accreditation. Reconstruction settings were harmonized using acquisitions of a Hoffman 3D brain phantom ${ }^{47}$ (data not shown). Participants were asked to refrain from smoking and from eating or drinking (except water) 3 and $6 \mathrm{~h}$, respectively, prior to the PET/CT-scan. One hour before the scan, participants consumed $150 \mathrm{mg}$ carbidopa and $400 \mathrm{mg}$ entacapone to reduce the formation of radiolabelled $\left[{ }^{18} \mathrm{~F}\right]$-FDOPA metabolites $^{48,49}$. Immediately prior to the PET acquisition, a low-dose CT-scan of the brain $(110 / 120 \mathrm{kVp}$, $35 \mathrm{mAs}$ ) was acquired for attenuation correction purposes. Subsequently, $150 \mathrm{MBq}\left[{ }^{18} \mathrm{~F}\right]$-FDOPA was administered intravenously as a bolus, which was followed by a 90-min dynamic PET acquisition. Head movement was minimalised with a headrest and head strap, and participants were monitored throughout the PET acquisition so that any discomfort could be observed and addressed. Data were collected in list mode and histogrammed into 25 timeframes $(5 \times 1,3 \times 2,3 \times 3$, and $14 \times 5$ minute $(\mathrm{s}))$. PET data were reconstructed iteratively with resolution modelling and 2-mm full-width-at-half-maximum Gaussian smoothing filter with a voxel size of $2 \times 2 \times 2 \mathrm{~mm}^{3}$.

\section{Image processing}

Image processing was conducted by researchers who were blind to ASD status. The MRI- and PET-images (except for the first two frames) were rigidly co-registered to a single PET-frame acquired $7 \mathrm{~min}$ post-injection using Vinci (v4.83; Max Planck Institute for Neurological Research, Cologne, Germany) $)^{50,51}$, based on mutual information ${ }^{52}$, to spatially align the images and compensate for minor head motion. Head movement was quantified by taking the square root of the sum of movement in millimetres squared in $x, y$, and $z$ directions from the last PET frame relative to the reference frame. Prior to pharmacokinetic analysis, volumes of interest (cerebellum and striatum) were generated automatically using PVElab (v2.3; Neurobiology Research Unit, Copenhagen, Denmark $)^{53,54}$ based on a maximum probability atlas ${ }^{55}$. In addition, SPM12 (Wellcome Centre for Human Neuroimaging, London, UK) was used to segment the MRI image into grey (GM) and white matter (WM). Subsequently, the GM cerebellum was used as a reference region to calculate the influx constant $k_{\mathrm{i}}^{\text {cer }}\left(\mathrm{min}^{-1}\right.$; from here on labelled as $\left.k_{\mathrm{i}}^{\text {cer }}\right)$ as a measure of dopamine synthesis capacity using reference Patlak graphical analysis ${ }^{56}$ as implemented in PPET (Amsterdam UMC, Amsterdam, The Netherlands) ${ }^{57}$. PET frames acquired between minutes 25 and 90 were used for linear fit, resulting in a whole-brain parametric image. From this parametric image, $k_{\mathrm{i}}^{\text {cer }}$ for the GM striatum was extracted.

The functional striatal sub-regions (associative, limbic, and sensorimotor), defined in the Oxford-GSK-Imanova brain atlas ${ }^{58}$, and a standard Montreal Neurological Imaging (MNI) brain template were extracted from FSLeyes v0.3 in FSL (v6.0; Analysis Group, Oxford, United Kingdom $)^{59,60}$. Using Vinci, the MNI-template was warped to match the participant MRI using a non-linear affine transformation. The resulting transformation 
matrix was applied to the striatal sub-regions, thereby warping them from standard- to subject-space. From the parametric image, we extracted GM $k_{\mathrm{i}}^{\text {cer }}$ values for voxels with at least $60 \%$ probability of belonging to the functional striatal sub-regions ${ }^{61}$.

We conducted several additional analyses to ensure the validity of the methods that we used (reported in Supplements 3.1-3.6). First, we calculated a whole-striatum $k_{\mathrm{i}}^{\text {cer }}$ value obtained from the three sub-regions weighted by their respective volumes and compared this to the $k_{\mathrm{i}}^{\text {cer }}$ value for the striatum obtained from the maximum probability atlas. Second, we repeated our main analyses with $k_{\mathrm{i}}^{\text {cer }}$ values extracted from the combined GM and WM tissue, since previous studies examining dopamine synthesis capacity did not always separate these tissues. Third, in the analyses with the striatal sub-regions, we additionally investigated a stricter threshold of at least 90\% probability. Fourth, we compared the mean standardized uptake values (SUV) in GM cerebellum in ASD and controls, to examine whether non-specific uptake of $\left[{ }^{18} \mathrm{~F}\right]$-FDOPA differed between groups. Finally, we repeated the main analyses for the two PET/CT-scanners separately.

\section{Statistical analyses}

Statistical analyses were conducted using SPSS v26. A two-tailed alpha of $\alpha=0.05$ was used to evaluate statistical significance. Statistical test assumptions were adequately met, unless stated otherwise. We had few missing data on the measures of social defeat (less than $0.25 \%$ of total responses on any questionnaire). Since imputation of these missing data did not change the outcomes of the study, we report the results without these data imputed.

For the primary analyses, we compared the striatal $k_{\mathrm{i}}^{\text {cer }}$ between ASD and controls using ANCOVA. We examined the relationship between striatal $k_{\mathrm{i}}^{\mathrm{cer}}$ and loneliness within ASD by conducting linear regression analysis. Analyses were adjusted for age, sex, smoking-status, and scanner-type (i.e., Biograph Horizon or Vereos) (for unadjusted results, see Supplement 4.1).

In the secondary analyses, group differences in social defeat were examined using independent-samples $t$-tests or $\chi^{2}$-tests. We report these results without adjusting for covariates, but regression analyses adjusted for age, sex, and smoking-status are reported in Supplement 4.2. The measures of childhood trauma and total bullying duration violated assumptions of normality and equality of variances, which we resolved by computing bootstrapped $p$ values $^{62,63}$. Furthermore, we conducted regression analyses to examine relationships between measures of social defeat (besides loneliness) and striatal $k_{\mathrm{i}}^{\mathrm{cer}}$. Finally, we conducted the previously-described analyses using the $k_{\mathrm{i}}^{\mathrm{cer}}$ in the three striatal sub-regions (rather than whole striatum) as dependent variables.

\section{Results}

\section{Participants}

Forty-five adults with ASD and 24 controls completed the study. For technical reasons, we were unable to use the data of three participants ( $n=1$ ASD, $n=2$ controls). Thus, the final sample included 44 adults with ASD and 22 controls. Table 1 shows the characteristics of the two samples. Average head movement was comparable in ASD and controls. We included 3 participants with substantial head movement $(>5 \mathrm{~mm} ; 1$ control and 2 ASD participants), as we excluded deviating frames during parametric analysis and the $k_{\mathrm{i}}^{\text {cer }}$ values of these participants fell within the range of values of the other participants. Moreover, excluding them did not alter the results.

\section{Dopamine synthesis capacity in ASD and controls}

Figure 1 shows the dopamine synthesis capacity in the striatum of adults with ASD and controls. In contrast to hypothesis 1a, we found no significant differences in dopamine synthesis capacity in ASD $(M=0.0145, S D=$ $0.0023)$ and controls $(M=0.0143, S D=0.0024)$ in the striatum after adjusting for covariates $\left(F_{1,60}=0.026, p=\right.$ 0.87). Moreover, as shown in Fig. 2, secondary analyses showed no significant group differences in dopamine synthesis capacity in the associative (ASD: $M=0.0155$, $S D=0.0024$; controls: $M=0.0155, S D=0.0027 ; F_{1,60}=$ $0.003, p=0.96$ ), limbic (ASD: $M=0.0152, S D=0.0026$; controls: $M=0.0151, \quad S D=0.0028 ; \quad F_{1,60}=0.000, p>$ 0.99 ), or sensorimotor (ASD: $M=0.0165, S D=0.030$; controls: $M=0.0166, S D=0.0032 ; \quad F_{1,60}=0.011, p=$ $0.92)$ striatal sub-regions after adjusting for covariates.

\section{Association between loneliness and dopamine synthesis capacity in ASD}

In contrast to hypothesis $1 \mathrm{~b}$, among adults with ASD there was no statistically significant association between

\section{Table 1 Sample characteristics of adults with autism spectrum disorder (ASD) and controls.}

\begin{tabular}{lrc}
\hline Variable & ASD $(\boldsymbol{n}=\mathbf{4 4})$ & Controls $(\boldsymbol{n}=\mathbf{2 2})$ \\
\hline Male, No. (\%) & $28(64 \%)$ & $14(64 \%)$ \\
Age in years, mean (SD) & $23.74(2.64)$ & $23.47(2.48)$ \\
Smoker, No. (\%) & $2(5 \%)$ & $1(5 \%)$ \\
Scanned on Vereos PET/CT-scanner, & $31(70 \%)$ & $13(59 \%)$ \\
No. (\%) & & \\
IQ, mean (SD) & $103.75(5.19)$ & $105.05(4.90)$ \\
Approximate injected [ ${ }^{18}$ F]-FDOPA & $161.55(7.26)$ & $157.24(8.57)$ \\
dose in MBq, mean (SD) & & \\
Head movement in millimetres, & $2.44(2.78)$ & $2.54(1.67)$ \\
mean (SD) & & \\
\hline
\end{tabular}

$S D$ standard deviation, $I Q$ intelligence quotient, $M B q$ megabecquerel. 


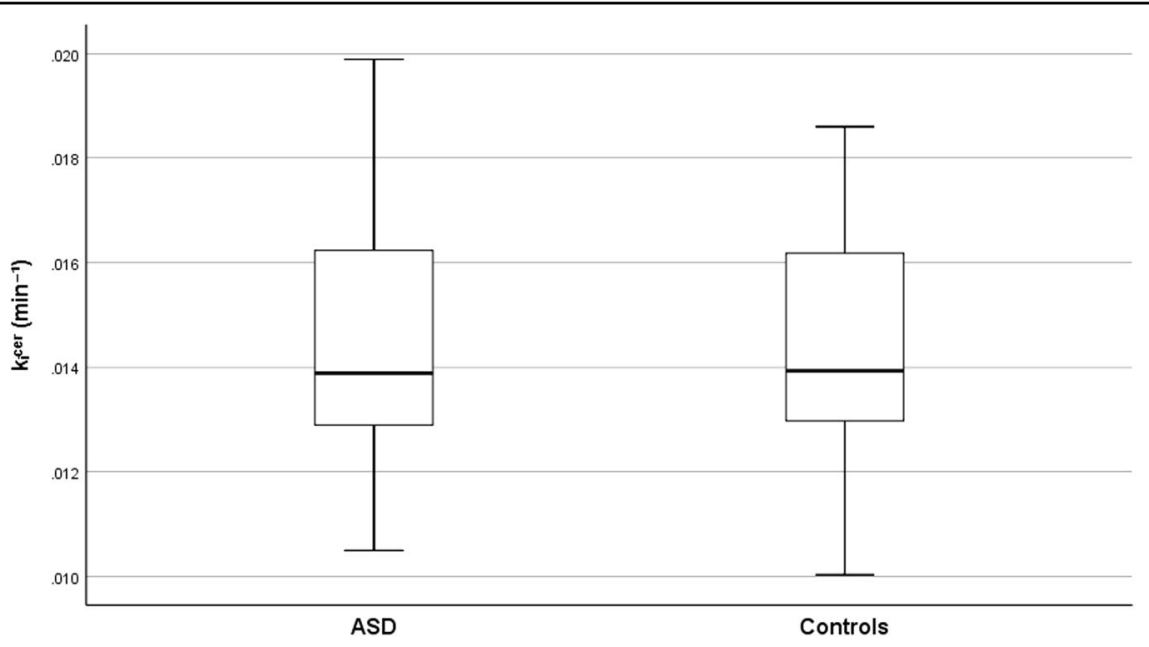

Fig. 1 Striatal dopamine synthesis capacity in adults with autism spectrum disorder (ASD) and controls. Boxplots show the median, quartiles, and range of striatal presynaptic dopamine synthesis capacity $\left(k_{i}^{c e r} \min ^{-1}\right)$.

loneliness and dopamine synthesis capacity in the striatum after adjusting for covariates $(\beta=0.01, p=0.96)$ (see Fig. 3). Furthermore, secondary analyses showed no significant associations between loneliness and dopamine synthesis capacity in the associative $(\beta=-0.03, p=0.87)$, limbic $(\beta=-0.01, p=0.93)$, or sensorimotor $(\beta=0.01$, $p=0.97)$ striatal sub-regions after adjusting for covariates.

\section{Secondary analyses with social defeat}

Adults with ASD reported more loneliness, more experiences of being ostracized, more and longer-lasting episodes of bullying victimization, smaller social networks, and more traumatic experiences in childhood than controls (see Table 2). Both samples reported a similar need to belong, but adults with ASD reported a lower availability of interpersonal support.

The relations between the secondary measures of social defeat and striatal dopamine synthesis capacity in adults with ASD are shown in Supplement 4.3. We found no significant association between the measures of social defeat with dopamine synthesis capacity in the striatum or any of its sub-regions after adjusting for covariates. A possible exception to this was childhood trauma (see Supplement 4.4), which did show a significant positive association with striatal dopamine synthesis capacity $(\beta=$ $0.32, p=0.05)$. However, this association was largely driven by one outlier (after excluding, $\beta=0.20, p=0.25$ ) and became non-significant after adjusting the $p$-value for multiple testing.

Notably, contrary to our expectations, exploratory posthoc analyses in controls showed a significant negative association between childhood trauma and striatal dopamine synthesis capacity (Supplement 4.5 and 4.6). On the other hand, the relationship between social support and dopamine synthesis capacity was negative in controls with a high need to belong (but positive in those with a low need to belong; Supplement 4.7).

\section{Associations with symptoms}

Adults with ASD $(M=3.13, S D=1.93)$ reported more psychotic symptoms on the PQ-16 than controls $(M=$ $1.06, S D=0.97)$. However, these symptoms were not significantly associated with dopamine synthesis capacity in the striatum in $\operatorname{ASD}(\beta=-0.05, p=0.81)$ or in controls $(\beta=-0.26, p=0.30)$ after adjusting for covariates. Results were also non-significant for the striatal subregions (see Supplement 4.8).

Exploratory analyses showed no statistically significant associations between dopamine synthesis capacity and depressed mood or anxiety (see Supplement 4.9).

\section{Sensitivity analyses}

As shown throughout Supplement $3, k_{\mathrm{i}}^{\text {cer }}$ values somewhat differed depending on the image processing procedure and/or PET/CT-scanner that was used, but the results of the analyses were comparable and conclusions remained unchanged. Non-specific uptake of $\left[{ }^{18} \mathrm{~F}\right]$ FDOPA in the cerebellum was similar in ASD and controls.

\section{Discussion}

We used PET/CT to examine striatal dopamine synthesis capacity in unmedicated, non-psychotic adults with ASD and its association with measures of social defeat. Contrary to our expectations, adults with ASD did not have a greater presynaptic dopamine synthesis capacity in the striatum or any of its sub-regions relative to controls. Furthermore, among adults with ASD we found no 

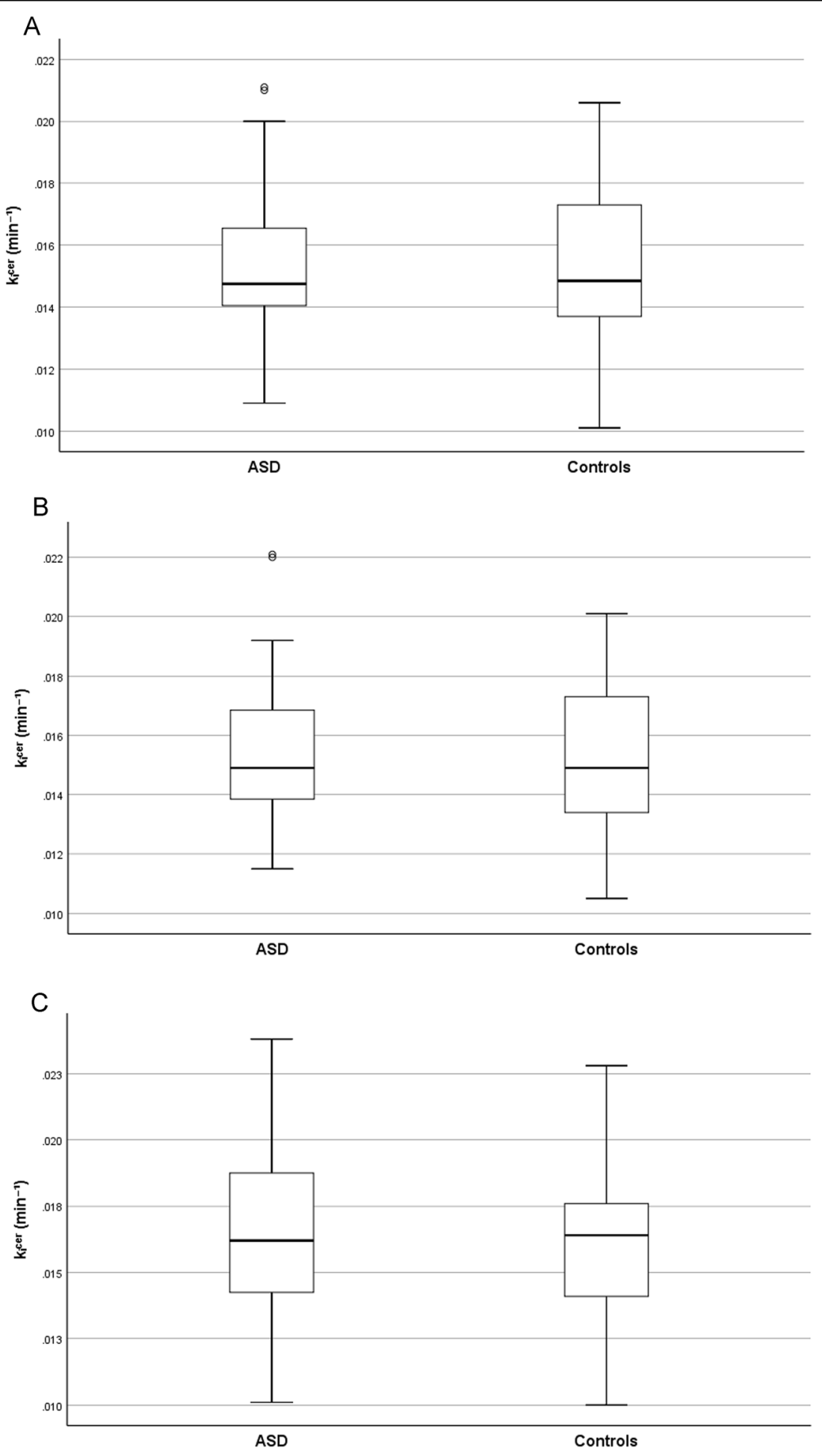

Fig. 2 Dopamine synthesis capacity in striatal sub-regions in adults with autism spectrum disorder (ASD) and controls. Boxplots show the median, quartiles, and range of presynaptic dopamine synthesis capacity $\left(k_{i}^{\mathrm{cer}} \mathrm{min}^{-1}\right)$ in the $\mathbf{A}$ associative, $\mathbf{B}$ limbic, and $\mathbf{C}$ sensorimotor striatum.

significant association between striatal dopamine synthesis capacity and loneliness or other measures of social defeat.
Our findings do not support previous ideas about the involvement of presynaptic dopamine signalling in $\mathrm{ASD}^{8}$. However, dopamine synthesis capacity is only one aspect 


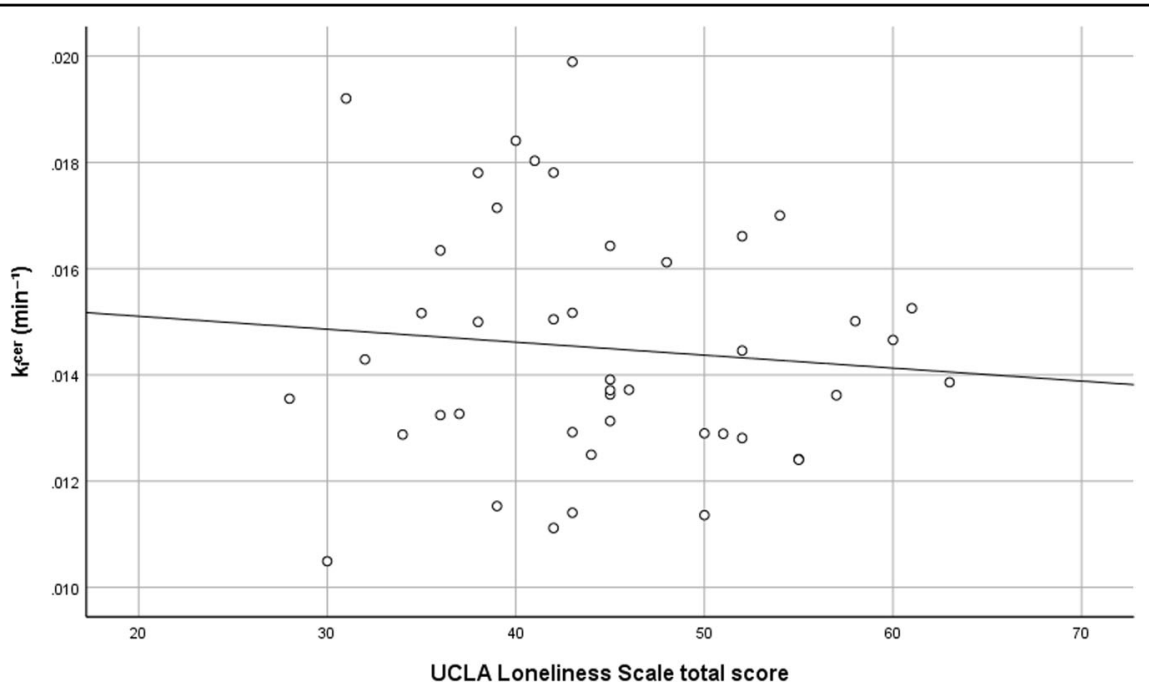

Fig. 3 Association between loneliness and striatal dopamine synthesis capacity in adults with autism spectrum disorder (ASD). Scatterplot shows the unadjusted relationship between total scores on the UCLA Loneliness Scale and presynaptic dopamine synthesis capacity $\left(k_{i}^{c e r} m_{i n}^{-1}\right)$ in the whole striatum.

Table 2 Measures of social defeat in adults with autism spectrum disorder (ASD) and controls.

\begin{tabular}{lccc}
\hline Variable & ASD $(\boldsymbol{n}=\mathbf{4 4})$ & Controls $(\boldsymbol{n}=\mathbf{2 2})$ & $\boldsymbol{p}$-value \\
\hline UCLA Loneliness Scale, mean (SD) & $44.66(8.65)$ & $32.14(5.77)$ & $p<0.001^{\mathrm{a}}$ \\
OES, mean (SD) & $29.73(5.76)$ & $21.86(4.35)$ & $p<0.001^{\mathrm{a}}$ \\
Bullied (yes/no), No. (\%) & $36(82 \%)$ & $11(50 \%)$ & $p=0.01^{\mathrm{d}}$ \\
Bullying total duration in months, mean (SD) & $48.57(40.57)$ & $11.32(18.63)$ & $p<0.001^{\mathrm{a}, \mathrm{b}, \mathrm{c}}$ \\
LSNS, mean (SD) & $15.89(5.34)$ & $21.36(3.13)$ & $p<0.001^{\mathrm{a}, \mathrm{b}}$ \\
CTQ, mean (SD) & $38.70(12.29)$ & $30.32(4.65)$ & $p<0.001^{\mathrm{a}, \mathrm{b}, \mathrm{c}}$ \\
NBS, mean (SD) & $31.36(6.43)$ & $31.45(4.62)$ & $p=0.95^{\mathrm{a}, \mathrm{b}}$ \\
ISEL, mean (SD) & $119.25(16.13)$ & $137.45(7.79)$ & $p<0.001^{\mathrm{a}, \mathrm{b}}$ \\
\hline
\end{tabular}

SD standard deviation, OES Ostracism Experience Scale, LSNS Lubben Social Network Scale, CTQ Childhood Trauma questionnaire, NBS Need to Belong Scale, ISEL Interpersonal Support Evaluation List.

andependent-samples $t$-test.

bqual variances not assumed.

'Estimated with 10000 bootstrapping samples.

${ }^{\mathrm{d}} \mathrm{X}^{2}$-test.

of dopamine signalling and other studies in ASD have found local alterations in dopamine transporter binding ${ }^{64}$ and dopamine receptor density ${ }^{65}$. Moreover, it is wellestablished that ASD is heterogeneous ${ }^{66}$, and as a result, deviations in dopamine synthesis might be present only in a subgroup of individuals. ASD adults with a low IQ, who used medication, and/or who had substantial psychotic symptoms were excluded from our study, and it would be interesting to examine whether abnormalities in dopamine synthesis capacity are present among them. Future studies can further elucidate whether alterations in dopamine functioning are involved in ASD.

The social defeat hypothesis of schizophrenia, which predicts that non-psychotic adults with ASD show an increase in dopamine synthesis capacity and that this capacity is associated with measures of social defeat ${ }^{19}$, was not supported. This might be explained as follows. First, the social defeat hypothesis might be incorrect. However, studies that examined striatal dopamine synthesis capacity and/or release in immigrants ${ }^{23}$, individuals with severe hearing impairment ${ }^{20}$, and individuals exposed to childhood trauma ${ }^{21,22}$ supported this hypothesis. Furthermore, experiments reported increased dopamine release in the nucleus accumbens of (sub)chronically defeated rodents ${ }^{67}$. In contrast, in line with post-hoc analyses with reference to childhood trauma in our control group, two recent studies observed negative associations between 
different types of social adversity and dopamine synthesis capacity ${ }^{68}$ or amphetamine-induced striatal dopamine release ${ }^{69}$. One possibility is that social defeat only increases dopamine functioning in certain individuals and that, for example, individuals with ASD respond neurochemically different to defeat than other risk groups. Nevertheless, given the high rates of social defeat in adults with ASD and the absence of a relationship with presynaptic dopamine, one can conclude from this study that social defeat per se does not necessarily upregulate dopamine synthesis capacity.

Second, dopamine sensitization might have been demonstrated if presynaptic dopamine release had been examined, for instance after a challenge with amphetamine. Since knowledge about the relationship between presynaptic dopamine synthesis capacity and amphetamine-induced dopamine release is limited, it is difficult to predict the outcome of this challenge ${ }^{70}$. To the best of our knowledge, presynaptic dopamine release has not yet been examined in ASD and it might be worthwhile to do so. It should be performed with great care, however, since inducing dopamine release might precipitate psychotic symptoms.

This study has several strengths. First, this is by far the largest PET/CT-study to examine dopamine synthesis capacity in ASD and the first to examine an association with possible risk factors. Given the (for PET/CT studies) large, a priori determined sample size, it is highly unlikely that the negative findings are due to a lack of statistical power. Second, we had strict inclusion measures, which might limit the generalizability of the findings to the entire ASD population but does reduce the influence of external factors such as (dopaminergic) medication use. Third, in addition to our main analysis with loneliness, we used multiple measures to assess social defeat, which consistently showed an absence of relation with striatal dopamine functioning.

The study also has several limitations. First, the study was cross-sectional. To understand how dopaminergic abnormalities arise, longitudinal studies are necessary. Second, we relied on self-report measures of social defeat, which may be prone to several types of bias. Third, PET/ CT-scans were acquired on two different PET/CT systems. However, reconstruction parameters for the two scanners were harmonized as much as possible and scanner-type was added as a covariate to the analyses. Moreover, conclusions did not change when we conducted the main analyses for the two PET/CT-scanners separately.

In conclusion, non-psychotic, unmedicated adults with ASD do not show a significant increase in striatal dopamine synthesis capacity, and this capacity is not associated with measures of social defeat.

\section{Acknowledgements}

We would like to thank Jacqueline Aanholt-Bijlemeer, Ina Boot, Neanke Bouwman, Robert Bovenkerk, Johan van Brecht, Michael Bruijns, Paul de Bruin, Mark van Buchem, Petra Dibbets-Schneider, Demi Jansen, Jordi Vonk-van Oosten, and Patrick van der Zwet for facilitating the MRI- and PET/CT-scans. Furthermore, we would like to express our gratitude to Daniëlle Bos, Carlijn Clemens, Truda Driesen, Deborah Op 't Eijnde, Erik Giltay, Jori Henke, and Jessie Kosterman for their assistance with conducting this study. We would also like to thank Ronald Boellaard, Patricia Cambraia Lopes, Elsmarieke van de Giessen, Sandeep Golla, Claus Svarer, and Charlotte van der Vos for their support with PET/CT data processing, and Fabian Termorshuizen for his support with the statistical analyses. Finally, we would like to thank JADOS (in particular Elles van Woerkum and Paul Stoffer), Anna Souverijn, Marcel Melchers, Els van der Ven, Villa Abel, PAS Nederland, Leviaan, aspergersyndroom.nl, autsider.net, RIAN Autismenetwerk, and the Dutch Association for Autism (Nederlandse Vereniging voor Autisme) for their help with recruiting study participants. The study was funded in part by Stichting J.M.C. Kapteinfonds.

\section{Author details}

${ }^{1}$ Rivierduinen Institute for Mental Healthcare, Leiden, The Netherlands. ${ }^{2}$ School for Mental Health and Neuroscience, Maastricht University, Maastricht, The Netherlands. ${ }^{3}$ Section of Nuclear Medicine, Department of Radiology, Leiden University Medical Center, Leiden, The Netherlands. ${ }^{4}$ Biomedical Imaging Group, University of Twente, Enschede, The Netherlands. ${ }^{5}$ Department of Radiology and Nuclear Medicine, Amsterdam University Medical Centers, location Vrije Universiteit Medical Center, Amsterdam, The Netherlands. ${ }^{6}$ Department of Radiology and Nuclear Medicine, Amsterdam University Medical Centers, location Academic Medical Center, Amsterdam, The Netherlands

\section{Conflict of interest}

The authors declare that they have no conflict of interest.

\section{Publisher's note}

Springer Nature remains neutral with regard to jurisdictional claims in published maps and institutional affiliations.

Supplementary Information accompanies this paper at (https://doi.org/ 10.1038/s41398-020-01174-w).

Received: 14 September 2020 Revised: 14 December 2020 Accepted: 15 December 2020

Published online: 13 January 2021

\section{References}

1. Lai, M.-C. et al. Prevalence of co-occurring mental health diagnoses in the autism population: a systematic review and meta-analysis. Lancet Psychiatry 6, 819-829 (2019).

2. Chisholm, K., Lin, A., Abu-Akel, A. \& Wood, S. J. The association between autism and schizophrenia spectrum disorders: A review of eight alternate models of co-occurrence. Neurosci. Biobehav. Rev. 55, 173-183 (2015).

3. McCutcheon, R., Beck, K., Jauhar, S. \& Howes, O. D. Defining the locus of dopaminergic dysfunction in schizophrenia: a meta-analysis and test of the mesolimbic hypothesis. Schizophr. Bull. 44, 1301-1311 (2018).

4. Howes, O. D. et al. Elevated striatal dopamine function linked to prodromal signs of schizophrenia. Arch. Gen. Psychiatry 66, 13-20 (2009).

5. Egerton, A. et al. Presynaptic striatal dopamine dysfunction in people at ultra-high risk for psychosis: findings in a second cohort. Biol. Psychiatry 74, 106-112 (2013)

6. Jauhar, S. et al. A test of the transdiagnostic dopamine hypothesis of psychosis using positron emission tomographic imaging in bipolar affective disorder and schizophrenia. JAMA Psychiatry 74, 1206-1213 (2017).

7. Marotta, R. et al. The neurochemistry of autism. Brain Sci. 10, 163 (2020).

8. Pavăl, D. A dopamine hypothesis of autism spectrum disorder. Dev. Neurosci. 39, 355-360 (2017).

9. Staal, W. G., Langen, M., Van Dijk, S., Mensen, V. T. \& Durston, S. DRD3 gene and striatum in autism spectrum disorder. Br. J. Psychiatry 206, 431-432 (2015). 
10. Oswald, D. P. \& Sonenklar, N. A. Medication use among children with autism spectrum disorders. J. Child Adolesc. Psychopharmacol. 17, 348-355 (2007).

11. Buck, T. R. et al. Psychiatric comorbidity and medication use in adults with autism spectrum disorder. J. Autism Dev. Disord. 44, 3063-3071 (2014).

12. Zürcher, N. R., Bhanot, A., McDougle, C. J. \& Hooker, J. M. A systematic review of molecular imaging (PET and SPECT) in autism spectrum disorder: current state and future research opportunities. Neurosci. Biobehav. Rev. 52, 56-73 (2015).

13. Ernst, M., Zametkin, A., Matochik, J., Pascualvaca, D. \& Cohen, R. Low medial prefrontal dopaminergic activity in autistic children. Lancet 350, 638 (1997).

14. Pain, L., Gobaille, S., Schleef, C., Aunis, D. \& Oberling, P. In vivo dopamine measurements in the nucleus accumbens after nonanesthetic and anesthetic doses of propofol in rats. Anesth. Analg. 95, 915-919 (2002).

15. Nieminen-von Wendt, T. S. et al. Increased presynaptic dopamine function in Asperger syndrome. NeuroReport 15, 757-760 (2004).

16. Mizrahi, R. Social stress and psychosis risk: common neurochemical substrates? Neuropsychopharmacology 41, 666-674 (2016).

17. Selten, J.-P., Booij, J., Buwalda, B. \& Meyer-Lindenberg, A. Biological mechanisms whereby social exclusion may contribute to the etiology of psychosis: a narrative review. Schizophr. Bull. 43, 287-292 (2017).

18. Selten, J.-P. \& Cantor-Graae, E. Social defeat: risk factor for schizophrenia? Br. J. Psychiatry 187, 101-102 (2005).

19. Selten, J.-P., van der Ven, E., Rutten, B. P. \& Cantor-Graae, E. The social defeat hypothesis of schizophrenia: an update. Schizophr. Bull. 39, 1180-1186 (2013).

20. Gevonden, M. et al. Increased release of dopamine in the striata of young adults with hearing impairment and its relevance for the social defeat hypothesis of schizophrenia. JAMA Psychiatry 71, 1364-1372 (2014).

21. Oswald, L. M. et al. History of childhood adversity is positively associated with ventral striatal dopamine responses to amphetamine. Psychopharmacology 231, 2417-2433 (2014).

22. Egerton, A. et al. Adversity in childhood linked to elevated striatal dopamine function in adulthood. Schizophr. Res. 176, 171-176 (2016).

23. Egerton, A. et al. Elevated striatal dopamine function in immigrants and their children: a risk mechanism for psychosis. Schizophr. Bull. 43, 293-301 (2017).

24. Bauminger, N., Shulman, C. \& Agam, G. Peer interaction and loneliness in highfunctioning children with autism. J. Autism Dev. Disord. 33, 489-507 (2003).

25. Sterzing, P. R., Shattuck, P. T., Narendorf, S. C., Wagner, M. \& Cooper, B. P. Bullying involvement and autism spectrum disorders: prevalence and correlates of bullying involvement among adolescents with an autism spectrum disorder. Arch. Pediatr. Adolesc. Med. 166, 1058-1064 (2012).

26. Schroeder, J. H., Cappadocia, M. C., Bebko, J. M., Pepler, D. J. \& Weiss, J. A. Shedding light on a pervasive problem: a review of research on bullying experiences among children with autism spectrum disorders. J. Autism Dev. Disord. 44, 1520-1534 (2014).

27. Johnson, T. D. \& Joshi, A. Dark clouds or silver linings? A stigma threat perspective on the implications of an autism diagnosis for workplace well-being. J. Appl. Psychol. 101, 430 (2016).

28. Hus, V. \& Lord, C. The autism diagnostic observation schedule, module 4: revised algorithm and standardized severity scores. J. Autism Dev. Disord. 44, 1996-2012 (2014).

29. Lord C. et al. Autism Diagnostic Observation Schedule, 2nd edn (Western Psychological Services, Los Angeles, 2012).

30. Yung, A. R. et al. Mapping the onset of psychosis: the comprehensive assessment of at-risk mental states. Aust. N. Z. J. Psychiatry 39, 964-971 (2005).

31. Ising, H. K. et al. The validity of the 16-item version of the Prodromal Questionnaire (PQ-16) to screen for ultra high risk of developing psychosis in the general help-seeking population. Schizophr. Bull. 38, 1288-1296 (2012).

32. Schmand, B., Bakker, D., Saan, R. \& Louman, J. The Dutch Reading Test for Adults: a measure of premorbid intelligence level. Tijdschr. Gerontol. Geriatr. 22, 15-19 (1991).

33. Ter Smitten R., Smeets R. \& Van den Brink W. Composite International Diagnostic Interview version 2.1: Dutch translation and adaptation (WHO-CIDI Training en Referentie Centrum voor het Nederlands taalgebied, Psychiatrisch Centrum AMC, Amsterdam, 1998).

34. Faul, F., Erdfelder, E., Lang, A.-G. \& Buchner, A. G* Power 3: A flexible statistical power analysis program for the social, behavioral, and biomedical sciences. Behav. Res. Methods 39, 175-191 (2007).

35. Faul, F., Erdfelder, E., Buchner, A. \& Lang, A.-G. Statistical power analyses using $\mathrm{G}^{*}$ Power 3.1: Tests for correlation and regression analyses. Behav. Res. Methods 41, 1149-1160 (2009).
36. Russell, D. W. UCLA Loneliness Scale (Version 3): reliability, validity, and factor structure. J. Pers. Assess. 66, 20-40 (1996).

37. Gilman, R., Carter-Sowell, A., DeWall, C. N., Adams, R. E. \& Carboni, I. Validation of the ostracism experience scale for adolescents. Psychol. Assess. 25, 319 (2013).

38. Olweus, D. The Revised Olweus BullyN Nictim Questionnaire (Research Center for Health Promotion of the University of Bergen, Bergen, 1996).

39. Lubben, J. et al. Performance of an abbreviated version of the Lubben Social Network Scale among three European community-dwelling older adult populations. Gerontologist 46, 503-513 (2006).

40. Bernstein, D. P. et al. Development and validation of a brief screening version of the Childhood Trauma Questionnaire. Child Abus. Negl. 27, 169-190 (2003).

41. Thombs, B. D., Bernstein, D. P., Lobbestael, J. \& Arntz, A. A validation study of the Dutch Childhood Trauma Questionnaire-Short Form: factor structure, reliability, and known-groups validity. Child Abus. Negl. 33, 518-523 (2009).

42. Leary, M. R., Kelly, K. M., Cottrell, C. A. \& Schreindorfer, L. S. Construct validity of the need to belong scale: Mapping the nomological network. J. Pers. Assess. 95, 610-624 (2013)

43. Cohen S., Mermelstein R., Kamarck T. \& Hoberman H. M. in Social Support: Theory, Research and Applications (eds Sarason I. G. \& Sarason B. R.) (Martinus Nijhoff Publishers, Dordrecht, 1985).

44. Loewy, R. L., Bearden, C. E., Johnson, J. K., Raine, A. \& Cannon, T. D. The prodromal questionnaire $(\mathrm{PQ})$ : preliminary validation of a self-report screening measure for prodromal and psychotic syndromes. Schizophr. Res. 79, 117-125 (2005).

45. Beck A. T., Steer R. A., Brown G. K. Beck Depression Inventory, 2nd ed. (The Psychological Corporation, San Antonio, 1996).

46. Spielberger, C. D. Manual for the State-Trait Anxiety Inventory (Form Y). (Consulting Psychologists Press, Palo Alto, 1983).

47. Boellaard, R. et al. Feasibility of brain PET/CT harmonization-a multicenter, multivendor pilot study. J. Nucl. Med. 59, 286 (2018).

48. Hoffman, J. M. et al. The effects of carbidopa administration on 6-[18F] fluoroL-dopa kinetics in positron emission tomography. J. Nud. Med. 33, 1472-1477 (1992).

49. Sawle, $G$ et al. The effect of entacapone (OR-611) on brain [18F]-6-L-fluorodopa metabolism: Implications for levodopa therapy of Parkinson's disease. Neurology 44, 1292-1292 (1994).

50. Vollmar S. et al. VINCl-Volume Imaging in Neurological Research, CoRegistration and ROls included. In Forschung und wissenschaftliches Rechnen 2003 (eds Kremer K., Macho V.) (GWDG, Göttingen, 2004).

51. MPI for Metabolism Research. VINCl. http://vinci.sf.mpg.de/ (2020).

52. Čižek, J. et al. Fast and robust registration of PET and MR images of human brain. Neuroimage 22, 434-442 (2004).

53. Quarantelli, M. et al. Integrated software for the analysis of brain PET/SPECT studies with partial-volume-effect correction. J. Nucl. Med. 45, 192-201 (2004).

54. Svarer, C. et al. MR-based automatic delineation of volumes of interest in human brain PET images using probability maps. Neuroimage 24, 969-979 (2005).

55. Hammers, A. et al. Three-dimensional maximum probability atlas of the human brain, with particular reference to the temporal lobe. Hum. Brain. Mapp. 19, 224-247 (2003).

56. Patlak, C. S. \& Blasberg, R. G. Graphical evaluation of blood-to-brain transfer constants from multiple-time uptake data. Generalizations. J. Cereb. Blood Flow. Metab. 5, 584-590 (1985).

57. Boellaard, R., Yaqub, M., Lubberink, M. \& Lammertsma, A. PPET: a software tool for kinetic and parametric analyses of dynamic PET studies. Neuroimage $\mathbf{3 1}$, T62 (2006).

58. Tziortzi, A. C. et al. Connectivity-based functional analysis of dopamine release in the striatum using diffusion-weighted MRI and positron emission tomography. Cereb. Cortex 24, 1165-1177 (2014).

59. Jenkinson, M., Beckmann, C. F., Behrens, T. E., Woolrich, M. W. \& Smith, S. M. Fsl. Neuroimage 62, 782-790 (2012).

60. McCarthy P. FSLeyes. https://users.fmrib.ox.ac.uk/paulmc/fsleyes/userdoc/ latest/index.html (2019).

61. Avram, M. et al. Reduced striatal dopamine synthesis capacity in patients with schizophrenia during remission of positive symptoms. Brain 142, 1813-1826 (2019).

62. Dwivedi, A. K., Mallawaarachchi, I. \& Alvarado, L. A. Analysis of small sample size studies using nonparametric bootstrap test with pooled resampling method. Stat. Med. 36, 2187-2205 (2017). 
63. Rietveld, T. \& van Hout, R. The $t$ test and beyond: recommendations for testing the central tendencies of two independent samples in research on speech, language and hearing pathology. J. Commun. Disord. 58, 158-168 (2015).

64. Nakamura, K. et al. Brain serotonin and dopamine transporter bindings in adults with high-functioning autism. Arch. Gen. Psychiatry 67, 59-68 (2010).

65. Kubota, M. et al. Binding of dopamine D1 receptor and noradrenaline transporter in individuals with autism spectrum disorder: A PET Study. Cereb. Cortex 30, 6458-6468 (2020).

66. Mottron L. \& Bzdok D. Autism spectrum heterogeneity: fact or artifact? Mol. Psychiatry 25, 3178-3185 (2020).
67. Hammels, C. et al. Defeat stress in rodents: from behavior to molecules. Neurosci. Biobehav. Rev. 59, 111-140 (2015).

68. Bloomfield, M. A., McCutcheon, R. A., Kempton, M., Freeman, T. P. \& Howes, O. The effects of psychosocial stress on dopaminergic function and the acute stress response. Elife 8, e46797 (2019).

69. Dahoun, T. et al. The relationship between childhood trauma, dopamine release and dexamphetamine-induced positive psychotic symptoms: a [11 C](+)-PHNO PET study. Transl. Psychiatry 9, 1-12 (2019).

70. Abi-Dargham, A., van de Giessen, E., Slifstein, M., Kegeles, L. S. \& Laruelle, M. Baseline and amphetamine-stimulated dopamine activity are related in drugnaive schizophrenic subjects. Biol. Psychiatry 65, 1091-1093 (2009). 